\title{
MECANISMOS DE PARTICIPACIÓN CIUDADANA EN EL SISTEMA DE EVALUACIÓN DE IMPACTO AMBIENTAL CHILENO
}

\author{
MarTín LARA \\ Universidad de Santiago de Chile \\ martin.felipe26@gmail.com \\ Diego Letelier \\ Universidad de Santiago de Chile \\ diegoletelier.araya@gmail.com
}

\section{RESUMEN}

Este artículo estudia la participación ciudadana en el marco del Sistema de Evaluación de Impacto Ambiental de Chile. Específicamente, se analiza el rol que han tenido los mecanismos de participación ciudadana dentro de este sistema. Con base en análisis estadísticos univariados y bivariados, se examinan 314 casos de proyectos de inversión mediados por algún grado de participación, ocurridos entre 2010 y 2015. Se evalúan diversas variables, tales como el sector productivo al que pertenecen, monto de la inversión, cantidad de observaciones y actividades del proceso de participación, entre otras. Se concluye que los procedimientos de evaluación de impacto ambiental presentan una baja participación de la ciudadanía, escasa cantidad de observaciones y actividades, pero, a la vez, ostentan un alto nivel de calificaciones favorables.

Palabras clave: Política ambiental, sistema de evaluación de impacto ambiental, participación ciudadana, Chile. 


\title{
CITIZEN PARTICIPATION MECHANISMS WITHIN THE CHILEAN SYSTEM OF ENVIRONMENTAL IMPACT ASSESSMENT
}

\begin{abstract}
This article studies citizen participation in the framework of the Chilean System of Environmental Impact Assessment. In particular, it analyzes the role of citizen participation mechanisms within this system. Using univariate and bivariate statistical analysis, 314 cases of investment projects carried out between 2010 and 2015, mediated by some degree of participation, are examined. Several variables are evaluated, such as the productive sector they belong to, investment sum, number of objections and activities in the participation process, among others. It is concluded that the procedures of environmental impact assessment evidence low citizen participation, scant objections and activities, whilst, at the same time, registering high favorable scores.
\end{abstract}

Keywords: Environmental policy, System of environmental impact assessment, Citizen participation, Chile. 


\section{INTRODUCCIÓN}

En Chile, desde el retorno a la democracia, existen dos hitos que han marcado el desarrollo de la política ambiental. En primer lugar, en 1994, la promulgación de la Ley 19.300 de Bases Generales del Medio Ambiente (LBGMA) y la creación de la Comisión Nacional del Medio Ambiente (Conama). En segundo lugar, en 2010, la promulgación de la Ley 20.417 que creó el Ministerio del Medio Ambiente, la Superintendencia del Medio Ambiente y el Servicio de Evaluación Ambiental (SEA). De esta manera, el SEA estaría destinado a administrar el Sistema de Evaluación de Impacto Ambiental (SEIA), creado en 1994, cuyo objetivo es evaluar todos aquellos proyectos de inversión públicos y privados en el país ${ }^{1}$.

En un principio, la LBGMA incorporó formas de participación ciudadana para los procesos de evaluación ambiental, a través de establecer acceso libre a la información y transparencia. Posteriormente, a partir de la reforma del 2010, el SEA se transformó en el principal responsable de llevar adelante estos procesos, permitiendo un involucramiento de la ciudadanía en la evaluación ambiental de los proyectos.

Desde una mirada teórica, dentro de los objetivos que persigue la aplicación de mecanismos de participación ciudadana en instancias de evaluación ambiental se encuentran: promover la cooperación entre el Estado y la ciudadanía, y prevenir y resolver conflictos de tipo ambiental. Bajo esta mirada, es posible establecer que en la medida de que existan procesos realmente efectivos de participación la tendencia de conflictos socioambientales debería disminuir (Mirosevic 2011).

No obstante, en Chile se ha podido observar que, tras la aprobación de ciertos proyectos de inversión, se han generado conflictos importantes con las distintas comunidades afectadas. Según Hernandez y Sazo (2015), del total de proyectos con conflictos socioambientales, entre 2000 y 2013, el $74 \%$ fue aprobado por la autoridad competente, mientras que apenas un $12 \%$ fue rechazado, revocado o suspendido. Estos datos sugieren que existe disconformidad social con la aprobación de determinados proyectos de inversión que afectan el medio ambiente.

Posiblemente, estas cifras contribuyen a evidenciar que los conflictos ambientales se han transformado en un problema de carácter político, el cual ha logrado incorporar con gran fuerza a diferentes actores de la sociedad (Estenssoro 2009).

1 Previo a la creación del SEA, el SEIA era dirigido de manera directa por la Conama. 
Siguiendo a dicha premisa, este artículo tiene por finalidad analizar los resultados de los mecanismos de participación ciudadana de la política ambiental en Chile desde 2010 hasta 2015.

En primer lugar, se aborda el concepto de participación ciudadana, en conjunto con algunos enfoques necesarios para el análisis posterior. En segundo lugar, se describe el proceso de evaluación ambiental y los mecanismos de participación que contempla la Ley. A continuación, se presentan los resultados identificados, con base en análisis estadísticos. Finalmente, se presenta un conjunto de conclusiones.

\section{CONCEPTO Y ENFOQUES DE LA PARTICIPACIÓN CIUDADANA}

Antes de precisar cualquier definición de participación ciudadana, primero es necesario desglosar este concepto en participación y ciudadanía. La participación se asume como un complejo proceso social, mediante el cual las personas se involucran en aspectos de la vida misma que son de su interés particular (Alarcón, Gómez y Rodríguez 2004). Montero (1996) define participación como una mutua transformación, donde el participante construye y modifica al objeto o hecho en el cual participa $y$, por el hecho de hacerlo, es también transformado. Lechner (1991) entiende la participación como la acción colectiva de los actores sociales e institucionales, de integrarse, proponer, controlar y evaluar críticamente los procesos de decisión pública en las esferas territoriales. Esta última, a diferencia de las anteriores, tiene una connotación más política y cercana a la definición de participación ciudadana que se abordará posteriormente.

Por otra parte, el concepto de ciudadanía puede ser entendido en constante relación con un proceso histórico fundamentalmente dinámico y reivindicativo. Por lo tanto, es un concepto en redefinición permanente (Vargas 1999). No obstante, siguiendo a Vargas, la ciudadanía se sustenta en rasgos comunes, como la igualdad formal de derechos y obligaciones, la pertenencia a una comunidad política (no solamente el Estado), la garantía para el ejercicio de los derechos ciudadanos vía instituciones ad hoc, la existencia de un espacio público más o menos desarrollado, entre otros. Finalmente, el Programa de las Naciones Unidas para el Desarrollo (PNUD 2014) se refiere a la ciudadanía en términos de sociedad civil, definiéndola como una esfera, creada históricamente, de derechos individuales y asociaciones voluntarias, en la que ocurre la concurrencia políticamente pacífica de unos con otros, en la persecución de sus respectivos asuntos, intereses e intenciones privadas, está garantizada por el Estado. 
Bajo esta premisa, la participación y la ciudadana se han transformado, en los últimos años, en una temática de suma relevancia para el debate de los procesos decisionales de las autoridades gubernamentales. Este protagonismo surge de la necesidad de generar mayor sustento, confianza y legitimidad en los distintos sistemas democráticos de cada país, por medio de la inclusión de la ciudadanía en los procesos de decisión en diferentes áreas de la intervención pública (Carmona 2012).

Con motivo de lo anterior, la participación ciudadana, como concepto, tiene su origen en los movimientos sociales (estudiantiles y obreros) de la década del sesenta en Estados Unidos, como respuesta a la necesidad que la ciudadanía tenía de incidir en las decisiones gubernamentales (Macpherson 2003). A partir de esta década, surge la idea de generar diversos espacios complementarios a la institucionalidad gubernamental tradicional, originándose así mecanismos de participación.

En este sentido, es posible decir que la participación ciudadana dibuja un escenario en el que las personas tienen la posibilidad de acceder al juego político, en aras de ajustar o reacomodar el espacio público en pro del bienestar colectivo (Alarcón, Gómez y Rodríguez 2004). Para Cunill (1991) y Llancar (2008), la participación corresponde a la intervención de los ciudadanos, en forma individual o colectiva, en el complejo y conflictivo proceso de la actividad pública, donde confluyen tanto intereses sociales como intereses del Estado. Por lo tanto, se puede establecer que la participación ciudadana es un medio que permite que el ciudadano forme parte de la definición, ejecución, control y evaluación de las decisiones de gobierno, siempre y cuando exista la voluntad del ciudadano de ejercer ese derecho.

En vista de lo señalado, el Estado debe velar por una ciudadanía más informada, que cada vez exige mayor acceso a la información, cumplimiento de mayores y mejores estándares y una incidencia concreta en las decisiones (Bermúdez 2014). Así, a raíz del empoderamiento de la ciudadanía, el PNUD (2014) ha constatado un aumento de la participación directa no institucionalizada en los últimos ańos en América Latina, que se manifiesta en marchas, movilizaciones, paralizaciones y huelgas, entre otras formas. Para explicar este fenómeno social, el PNUD propone dos hipótesis: En primer término, una hipótesis de polarización, la que establece que las protestas ciudadanas son el producto del distanciamiento entre la participación política, debido a los bajos niveles de legitimidad y altos niveles de conflictividad. Y, en segundo término, la hipótesis de normalización, la que establece que las protestas son una manera de incidir e influir en las decisiones colectivas y en las políticas públicas. 
En consecuencia, la falta de participación política es una de las causas directas de la carencia de legitimidad y de desconfianza que existe actualmente en la política y sus respectivas instituciones (Villarroel 2014). En la medida que no exista participación institucionalizada esta situación podría desencadenar un conflicto social. En este sentido, el objetivo del conflicto social es la restauración de una situación que se considera como injusta y que genera discordia en una de las partes, creando una nueva situación de equilibrio a través de la adecuación del derecho (Oro 2014). Dado lo anterior, la aplicación de mecanismos de participación ciudadana se asoma como una solución plausible; ya que tiende a prevenir y resolver conflictos, promoviendo una mayor cooperación entre el Estado y la ciudadanía.

Finalmente, tomando en cuenta las definiciones de participación ciudadana propuestas, se presenta el enfoque de matriz de participación ciudadana (IAP2 2007), que permitirá mejorar el entendimiento de los diferentes niveles de participación que defienden algunos autores. Este se diseñó identificando los distintos tipos de participación en relación a los propósitos, promesas, herramientas y técnicas que cada una de estas persigue. Este enfoque permite conocer cómo se desenvuelven estos niveles de participación, bajo ciertas variables transversales (Reyes y Ríos 2016). Mientras más se avanza en la matriz, mayor es el impacto que tienen los ciudadanos en el proceso de toma de decisión. 
En el primer nivel de la matriz, se encuentra el acceso a la información. Este no es considerado como una etapa participativa propiamente tal, debido a la unidireccionalidad de la relación, pero constituye el pilar en el que todo involucramiento del público debe basarse. En el segundo nivel, el público no solo recibe, sino que también entrega información, la que puede ser utilizada por los tomadores de decisiones para enriquecer las opciones de planes, políticas o proyectos a implementar. A diferencia del nivel anterior, el flujo de información es bidireccional: el público es un miembro activo. El tercer nivel de la matriz profundiza el grado de interacción con los ciudadanos, en particular, respecto a las alternativas a implementar. Así, se busca, en conjunto, generar distintas alternativas posibles de ser materializadas en la decisión final. En un cuarto nivel el trabajo se realiza entre los actores involucrados. Esto implica no solo la deliberación, sino también la búsqueda de consensos o acuerdos entre los participantes. Esta colaboración implica una relación que excede la mera negociación, donde los actores se unen para trabajar de manera coordinada en la implementación de políticas o planes específicos. Por último, en el quinto nivel la ciudadanía es quien tiene la última palabra en la toma de decisiones. Es decir, se garantiza el derecho a decir no.

Es preciso mencionar que todas estas categorías deben entenderse como un continuo, puesto que no pueden darse niveles más profundos de participación si no existen los anteriores (Reyes y Ríos 2016).

\section{METODOLOGÍA}

Para comprender los mecanismos de participación ciudadana del SEIA en Chile se privilegia un diseño de investigación fundamentalmente cuantitativo. En este marco, dado que aún es escasa la información teórica y empírica en materia medioambiental, se opta por el análisis descriptivo. En esta línea, se realizan análisis estadísticos univariados y bivariados de todos los casos de evaluación que contemplaron mecanismos de participación ciudadana ingresados al SEIA entre los ańos 2010 y 2015. Esto es complementado con una revisión crítica de fuentes primarias y secundarias, ejercicio que permite dar luces acerca de la incidencia que tienen los procesos de participación en la evaluación de estos proyectos.

Para el caso del análisis univariado, se utilizan instrumentos tales como distribuciones de frecuencia, representaciones gráficas y medidas de tendencia central. Por otro lado, en el análisis bivariado, se emplean pruebas de significación estadística, como el coeficiente de correlación de Pearson $(R)$ y coeficiente de determinación $\left(R^{2}\right)$. 
En relación a las fuentes de los datos recolectados, se recurrió directamente al SEA a través de una solicitud amparada por la Ley de Transparencia. De esta manera, se solicitaron todos aquellos proyectos que contemplaron algún proceso de participación entre el 1 de enero de 2010 y el 31 de diciembre de 2015. A partir de esto, se consolidó una muestra de 314 observaciones.

Asimismo, se seleccionaron nueve variables para el análisis descriptivo.

$\mathrm{X}_{\mathrm{I}}$ : Tipo de evaluación, declaración de impacto ambiental (DIA) o estudio de impacto ambiental (EIA).

$\mathrm{X}_{2}$ : Región.

$\mathrm{X}_{3}$ : Sector productivo.

$\mathrm{X}_{4}$ : Titular. Persona, natural o jurídica, mandante de realizar un proyecto de inversión en el país.

$\mathrm{X}_{5}$ : Estado de calificación. Proyectos aprobados, desistidos, no calificados, no admitidos a tramitación, revocados y rechazados.

$\mathrm{X}_{6}$ : Fecha de presentación del proyecto (ingreso al sistema).

$\mathrm{X}_{7}$ : Fecha de calificación (resolución).

$\mathrm{X}_{8}$ : Inversión del proyecto.

$\mathrm{X}_{9}$ : Tiempo de demora de la calificación.

\section{EL PROCEDIMIENTO DE EVALUACIÓN DE IMPACTO AMBIENTAL}

El SEA es un organismo público descentralizado y regionalmente desconcentrado - posee Direcciones Regionales de Evaluación Ambiental- con personalidad jurídica y patrimonio propio, sometido a la supervigilancia del Presidente de la República por medio del Ministerio del Medio Ambiente. Su creación, en 2010, responde al objetivo de establecer un organismo de gestión ambiental capaz de administrar el SEIA, en reemplazo de la Conama (Boettiger 2010).

El SEA se encuentra dirigido por un Director Ejecutivo (jefe de servicio) y por directores regionales. Adicionalmente, está regido por dos órganos colegiados: la Comisión de Evaluación ${ }^{2}$, encargada de calificar ambientalmente los proyectos sometidos a evaluación, y el Comité

2 Está integrada a nivel regional por los SEREMIs de Medio Ambiente, Salud, Economía, Energía, Obras Públicas, Agricultura, Vivienda y Urbanismo, Transportes y Telecomunicaciones, Minería y de Planificación (Desarrollo Social), y por último, el Intendente y el Director Regional del SEA, quien actúa como secretario. 
Técnico ${ }^{3}$, encargado de elaborar un acta de evaluación para cada proyecto, a modo de base para la calificación ambiental de la Comisión, la que deberá ser de libre acceso para el público (Artículo 86, Ley 19.300).

El SEIA se instituyó en 1994 con la promulgación de la Ley 19.300, como un proceso sistemático y global para evaluar ambientalmente los proyectos. Este estableció un modelo de ventanilla única para la obtención de permisos ambientales, teniendo un proceso global con plazos claros. Así, los proyectos que ingresan al SEIA solo pueden ser aprobados si cumplen con la normativa ambiental y se hacen cargo de todos sus impactos ambientales significativos.

En este sentido, el impacto se mide de dos formas. La primera es en un ámbito general, que se ciñe a la siguiente definición de medio ambiente:

El sistema global constituido por elementos naturales y artificiales de naturaleza física, química o biológica, socioculturales y sus interacciones, en permanente modificación por la acción humana o natural y que rige y condiciona la existencia y desarrollo de la vida en sus múltiples manifestaciones (Artículo 2, II), Ley 19.300).

La segunda forma de evaluar los impactos, responde a dimensiones más particulares de lo que se entiende por impacto ambiental -definidas por el artículo 11 de la Ley 19.300-, a saber: riesgo para la salud de la población, efectos adversos significativos sobre los recursos naturales renovables, localización en o próxima a poblaciones, recursos o áreas protegidas, entre otras.

Luego, lo primero es definir si el proyecto califica o no para ingresar al sistema. El artículo 3 del reglamento contempla una serie de actividades físicas detalladas para los cuales se establecen umbrales mínimos para esto, siendo los proyectos que generen carga ambiental, en cualquiera de sus fases, los que deberán someterse a evaluación. Si el proyecto no cumple con alguna de estas características, el titular solo debe obtener los permisos sectoriales correspondientes.

Lo siguiente es definir qué se presentará, una Declaración de Impacto Ambiental (DIA) o un Estudio de Impacto Ambiental (EIA). Los EIA son requeridos para proyectos que pueden generar cualquiera de los impactos

3 Se encuentra integrada por el SEREMI de Medio Ambiente, el Director Regional del SEA, los Directores Regionales de los servicios públicos con competencia ambiental, el Gobernador Marítimo y el Consejo de Monumentos Nacionales. 
significativos específicamente mencionados en el artículo 11 de la Ley 19.300, y consisten en documentos más detallados donde se evalúan esos impactos. Si no se generan estos impactos, el titular deberá elaborar un DIA.

Una vez determinado qué documento redactar, el reglamento especifica los contenidos mínimos que el EIA o el DIA deben tener. Cabe destacar que es el titular del proyecto quien identifica los impactos ambientales que su proyecto producirá, y el grado o alcance que cada uno de estos tiene, por lo que posee total autonomía en esta etapa.

Posteriormente, una vez redactado el tipo de documento, es ingresado al Sistema. Aquí los evaluadores pueden identificar carencias en el alcance del documento y exigir la ampliación de la información o el análisis. Para ello, el SEA distribuye el documento a todos los diferentes servicios públicos con competencia ambiental ${ }^{4}$. Para este proceso, las instituciones tienen un plazo $^{5}$ de 30 días en caso de Estudio y de 15 días en caso de Declaración, donde existe la posibilidad de que cada institución pueda comunicar su decisión de no participar.

Ahora bien, si cualquiera de los servicios, incluyendo al SEA, realizan observaciones al documento, estos serán compilados en un informe conocido como Informe Consolidado de Solicitud de Aclaraciones, Rectificaciones y/o Ampliaciones (ICSARA), documento que será enviado al titular y quien tendrá la obligación de responder a través de un documento denominado adenda. Esto inicia un proceso iterativo en el que las agencias pueden requerir más adendas mientras sigan teniendo observaciones. Sin embargo, con el nuevo reglamento se limitó esta exigencia a un máximo de dos, con la posibilidad de ampliar a una tercera de ser necesario y debidamente justificado.

Una vez acabado el proceso de adendas, el SEA emite el Informe Consolidado de Evaluación (ICE). Este resume la evaluación técnica y constituye el material a partir del cual el Comité de Evaluación tomará la decisión. Esta decisión será emitida formalmente mediante la Resolución

4 Según el artículo 24 del reglamento, estos están definidos como: “[...] aquellos que cuenten con atribuciones en materia de permisos o pronunciamientos ambientales sectoriales respecto del proyecto o actividad en particular" (Decreto Supremo 40, 2013)

5 Todo plazo contabilizado en el reglamento es en días hábiles (Artículo 23, Decreto Supremo 40, 2013). 
de Calificación Ambiental (RCA). Así, el plazo para el proceso general es de 180 días para un EIA y 90 para el DIA, los que no consideran los días en que el titular decide suspender el proceso para preparar las adendas. A pesar de ello, el artículo 45 del reglamento permite ampliar el plazo de evaluación en casos calificados y debidamente fundados, donde el Director Regional o el Director Ejecutivo del Servicio, según sea el caso, podrá (por una sola vez) ampliar el plazo definido anteriormente, hasta por 60 días adicionales en caso de EIA y de 30 en caso de DIA.

Por último, si el proyecto es calificado favorablemente, el titular requiere los permisos sectoriales o mixtos complementarios necesarios, especificados desde el artículo 111 al artículo 160 del reglamento, para poder comenzar a ejecutar su actividad. Finalmente, corresponderá a la Superintendencia del Medio Ambiente fiscalizar el cumplimiento de las condiciones, normas y medidas establecidas en la RCA de cada proyecto.

\section{MECANISMOS DE PARTICIPACIÓN ASOCIADOS AL PROCEDIMIENTO}

En el presente apartado se explican cuatro mecanismos de participación: 1) el acceso a la información; 2) el derecho a realizar observaciones; 3) el proceso de consulta indígena ${ }^{6}$; y 4) el proceso de reclamaciones. Las primeras tres se realizan durante el proceso técnico de evaluación y las reclamaciones se realizan para revisar la RCA. En otras palabras, las tres primeras son un mecanismo preventivo o ex ante y el último es ex post.

El primero, el acceso a la información, es un aspecto fundamental, puesto que permite el ejercicio de los derechos contemplados en la Constitución y las distintas normas que hacen alusión al medio ambiente y la transparencia. En la actualidad se exigen ciudadanos informados, con acceso a documentos, resoluciones, estudios y antecedentes en poder de las autoridades. En conformidad a la Ley 20.285 de Acceso a la Información Pública, la reforma a la Ley 19.300 logró importantes avances en esta materia. Según el artículo 31 bis de esta última, toda persona tiene derecho a acceder a la información de carácter ambiental que se encuentre en poder

6 El reglamento entiende como pueblo indígena lo estipulado en el inciso segundo del artículo 1 de la Ley 19.253 (1993). Esta reconoce como pueblo indígena a: Mapuche, Aimara, Rapa Nui, las comunidades Atacameñas, Quechuas, Collas y Diaguita del norte de Chile, las comunidades Kawashkar o Alacalufe y Yámana o Yagán de los canales australes. Por otra parte indígena, según lo que dice el artículo 2 de la Ley 19.253, el chileno hijo de padre o madre indígena, un descendiente con apellido indígena o los que mantengan rasgos culturales de alguna etnia indígena. 
de la administración.

En este sentido, dentro del proceso de evaluación, existen diferentes canales de flujo de información a los ciudadanos. Según el artículo 20 y 21 del reglamento, existe un expediente físico y electrónico de cualquier proyecto que se someta a evaluación en el SEIA, y los ciudadanos podrán acceder a él o solicitar, a su costa, reproducciones del expediente durante cualquier momento de la evaluación. Además, físicamente será exhibido en las dependencias de la municipalidad respectiva. Sin perjuicio de aquello, existe información que puede ser reservada a pedido del titular. Por otro lado, en el Diario Oficial y en un periódico de circulación regional o nacional, según sea el caso, se publican los extractos de los EIA, a costa del titular, y un listado de los DIA, publicado el primer día hábil del mes sobre los proyectos del mes inmediatamente anterior. Por último, el titular debe realizar avisos radiales, en una emisora local o regional, del proyecto en cuestión.

El segundo mecanismo que está en el reglamento es el derecho a realizar observaciones. Este consiste en una forma de canalizar las opiniones de los ciudadanos a través de un documento escrito que permite a la persona, natural o jurídica, realizar sus descargos o inquietudes sobre un proyecto determinado, con el requisito explícito de contener fundamentos técnicos y que se refiera a la evaluación ambiental de la actividad. Este derecho se ve extendido por el artículo 91 del reglamento, que estipula que las observaciones que cumplan con los requisitos serán evaluadas técnicamente, tendrán una respuesta fundada por el servicio y serán consideradas tanto en el ICE como en la RCA.

En relación a los EIA, una vez publicado el extracto en el diario, existe un plazo de 60 días hábiles para realizar observaciones. En el caso de la DIA, se debe abrir un procedimiento de participación ciudadana, donde el Director Regional o el Director Ejecutivo, según corresponda, abrirá un proceso de observaciones para aquellas que generen impactos ambientales en las comunidades próximas ${ }^{7}$.

Por último, en caso de que exista un proceso de participación asociado, el Servicio deberá propiciar instancias de información, con actividades de

7 Siempre y cuando, a lo menos dos organizaciones ciudadanas con personalidad jurídica o mínimo diez personas naturales directamente afectadas, lo soliciten, por escrito y durante los diez días siguientes a la publicación en el Diario Oficial del proyecto o actividad en cuestión. En este caso, el proceso tendrá una duración de veinte días. 
capacitación en materia ambiental o de encuentros entre el titular y la comunidad. Para ello, el servicio podrá solicitar la colaboración de órganos del Estado con competencia ambiental o con competencia en materia de desarrollo comunitario, social o indígena. Estas actividades serán de utilidad para acercar y explicar la normativa ambiental a los ciudadanos, para que hagan uso de los derechos que la Ley y el reglamento les confiere.

En tercer lugar, existe un proceso de consulta indígena que se asemeja al proceso ciudadano de observaciones. Este se da en el caso de que un proyecto o actividad genere algunos de los efectos indicados en los artículos 7, 8 y 10 del reglamento ${ }^{8}$ y afecte directamente a uno o más grupos humanos pertenecientes a grupos indígenas. Estas condiciones obligan al Servicio a diseñar y desarrollar un proceso de consulta de buena fe, que contemple mecanismos apropiados para que el grupo o grupos en cuestión puedan participar de manera informada y puedan influir durante el proceso de evaluación ambiental. Durante este proceso, los pueblos indígenas afectados participarán de manera exclusiva, con el objetivo de llegar a un acuerdo o lograr el consentimiento.

En cuarto lugar, las reclamaciones son un recurso que puede utilizar tanto el proponente como un ciudadano afectado. El titular puede proceder una reclamación ante el Director Ejecutivo o el Comité de Evaluación, según DIA o EIA respectivamente, en contra de la resolución que niegue lugar, rechace o establezca condiciones al proyecto. Este recurso puede ser interpuesto en un plazo de 30 días, contados una vez que la resolución fue notificada, y la autoridad competente tendrá 30 o 60 días, DIA o EIA respectivamente, para resolver la reclamación.

En cambio, la reclamación que puede interponer la ciudadanía es notablemente inferior, dentro de los mismos plazos. Solo las personas, naturales o jurídicas, que realizaron observaciones y que estas no hubieren sido consideradas en los fundamentos de la respectiva resolución, podrán presentar un recurso de reclamación. Si la reclamación es rechazada, es posible acudir al Tribunal Ambiental para impugnar esta decisión.

Cabe mencionar también, como un quinto elemento de análisis, que bajo el artículo 17 del reglamento existe la posibilidad de una negociación directa de compensación o mitigación ambiental entre el titular del proyecto-antes de que el proyecto sea ingresado o durante el proceso de

8 El artículo 7 se refiere al reasentamiento de comunidades humanas, o alteración de los sistemas de vida de los grupos humanos; el artículo 8 se refiere a la localización y valor ambiental del territorio; y por último, el artículo 10 del reglamento se refiere a la alteración del patrimonio cultural. 
evaluación-y las comunidades afectadas, situación que debe ser informada a la autoridad. Sin embargo, ante un eventual acuerdo, este no será vinculante para la calificación ambiental del proyecto o actividad, lo que resta incentivos al titular de realizarlo.

\section{ANÁLISIS CRÍTICO DE LA PARTICIPACIÓN EN LA INSTITUCIONALIDAD AMBIENTAL}

La problemática medioambiental ha sido parte de un proceso histórico de socialización de los organismos internacionales, que comienzan en la década del setenta y ochenta con la Conferencia del Medio Ambiente Humano de Estocolmo y la Comisión Mundial sobre el Medio Ambiente y el Desarrollo (CMMAD), que establecieron las directrices para una política medioambiental en Chile (Estenssoro 2009) ${ }^{9}$. Sin embargo, el verdadero impulsor para establecer las primeras bases institucionales de una política medioambiental acorde a la discusión internacional vigente fue la Cumbre de Río celebrada en 1992, cuya discusión tendía a compatibilizar los esfuerzos del crecimiento económico con la idea del desarrollo sostenible (Olivares 2010, Zelada y Park 2013).

Con este impulso, se promulgó la LBGMA, ley que tuvo un impacto significativo dentro de la temática ambiental en Chile, puesto que instauró una institucionalidad que aunó a los organismos con competencia ambiental bajo una misma tutela. Así, la Conama se convirtió en el primer organismo que intentó liderar la política ambiental chilena. Por otro lado, se dotó al Estado de distintos instrumentos de gestión ambiental, tales como el SEIA, programas de información ambiental, planes de prevención y áreas protegidas, entre otras.

Ahora bien, independiente de los logros alcanzados a nivel institucional, diversos actores del mundo social, académico y político fueron críticos a nivel de esta institucionalidad, especialmente por la falta de coordinación a nivel interno entre autoridades ambientales y sectoriales. Esta situación derivó en el uso y abuso de recursos de protección, mecanismo de defensa que utilizaron las comunidades en los conflictos ambientales (Sierra 2008). Asimismo, desde la entrada en vigencia de la LBGMA en 1994, se hizo notar que los mecanismos de evaluación de los proyectos del SEIA serían insuficientes para canalizar la creciente conflictividad ambiental y

9 Se establece en la Constitución Política de la República de 1980, en su artículo 19, número 8 , el derecho a vivir en un medio ambiente libre de contaminación, junto con la creación de la Comisión Nacional de Ecología en 1984, órgano encargado de proponer acciones sobre el medio ambiente. 
la consiguiente demanda por participación en las decisiones ambientales (Sepúlveda y Rojas 2010). No obstante, fue el informe de Evaluación de Desempeño Ambiental de la OCDE (2005) el verdadero impulso para establecer cambios a la política e institucionalidad instaurada inicialmente, de manera de modernizar el sistema y garantizar los derechos ciudadanos en materia medio ambiental.

Con base en estas diferentes causas, el 26 de enero del año 2010 se publica en el Diario Oficial la Ley No 20.417, que crea el Ministerio de Medio Ambiente, el Servicio de Evaluación Ambiental (SEA) y la Superintendencia de Medio Ambiente, modificando así la Ley No 19.300. Esta reforma logró consagrar el derecho al acceso a la información, con la introducción de una serie de sistemas de información ambiental (Bermúdez 2010).

A pesar de que la reforma de 2010 contribuyó a mejorar la calidad técnica, dejó de lado la capacidad de incidencia de las comunidades y grupos afectados en las decisiones ambientales (Berdichevsky y Sepúlveda 2016). En este sentido, no existió una alteración significativa al rol que los ciudadanos y las comunidades ocupan dentro del proceso, manteniendo algunos de los vicios que el sistema ya presentaba.

Ahora bien, para entender la profundidad de la participación, se debe tener en cuenta el grado de participación del público en la construcción de alternativas y en la toma final de decisiones (Reyes y Ríos 2016). El rol del Estado es crucial para asegurar que estas instancias permitan involucrar a todos los ciudadanos de manera amplia. De esta manera, para algunos autores la participación ciudadana sirve para educar y concienciar a la ciudadanía (Maldonado 2000), cuya definición es el reflejo del mecanismo de acceso a la información dentro del procedimiento. Como consecuencia de la reforma, los ciudadanos pueden acceder y solicitar información por varios canales de formales, lo que permite el libre flujo de información con respecto a los proyectos que se evalúan dentro del sistema.

Sin embargo, desde la perspectiva del ciudadano, existe una falta de capacidad técnica propia, o la dificultad de acceder a apoyo técnico de terceros, que termina siendo considerada una falencia dentro del sistema. Los EIA, en particular, son extensos y utilizan un lenguaje complejo, sus plazos legales son limitados, situación que condiciona la oportunidad de comprensión para cualquier ciudadano. En consecuencia, muchas personas no pueden acceder a la información. Finalmente, se coarta la participación activa. 
Ahora bien, en la literatura existe un consenso respecto a que el acceso a la información solo es un paso anterior al establecimiento de mecanismos que aseguren a los ciudadanos la influencia en las decisiones (Cunill 1991). La real participación es aquella donde los ciudadanos organizados intervienen en los procesos de actividad pública, y en los que se les permite ser parte de este juego político (Alarcón, Gómez y Rodríguez 2004). En conclusión, la disponibilidad de información para todo público es un requisito básico, pero no significa por sí solo que exista participación ciudadana. Entonces, es obligatorio que esta herramienta sea complementada con otros mecanismos para lograr los objetivos de la participación ciudadana. Para ello, el procedimiento cuenta con las observaciones, actividades y el proceso de consulta indígena explicados anteriormente. Si bien es cierto que la reforma realizó algunos cambios, esta no tuvo una profundidad notable, por lo que se han perpetuado algunas falencias del sistema.

En primer lugar, en términos de observaciones, el enfoque está centrado en los aspectos estrictamente técnicos. Esto significa que los ciudadanos deben pronunciarse sobre los impactos identificados en los respectivos EIAs o DIAs, lo que disminuye la posibilidad por parte de los ciudadanos de ampliar los impactos ya establecidos o de incidir en su jerarquización, lo que demuestra una potente diferencia de oportunidad con respecto al titular, quien propone y orienta estos impactos analizados. Por otro lado, estas observaciones deben ser fundadas, es decir, expresadas en términos que puedan ser procesadas por los técnicos encargados, lo que convierte a la participación ciudadana en un asunto cada vez más excluyente. Este aspecto perpetúa la diferencia de recursos y herramientas que existe entre el titular y la ciudadanía, dificultando una incidencia mayor (Berdichevsky y Sepúlveda 2016).

Para tratar de solventar estas diferencias el SEIA tiene como deber generar actividades de encuentro entre el titular y la ciudadanía y actividades de capacitación ambiental a las comunidades. Sin embargo, al revisar estos registros, se observa una asistencia limitada en comparación a la población total de las comunas o localidades donde se realiza la actividad, siendo los representantes de las comunidades afectadas los asistentes habituales (Berdichevsky y Selpúlveda 2016, Reyes y Ríos 2016). Sumado a lo anterior, el acotado plazo para los procesos de participación ciudadana genera un limitado espacio para maniobrar, y las actividades que buscan capacitar a las comunidades no logran tener el efecto necesario. En este sentido, existe un claro déficit de capacidades, tanto de las comunidades como de los ciudadanos para tomar parte en estas actividades, como de los organismos públicos para diseñarlas y conducirlas (Durán et al. 2016). 
Finalmente, el proceso de consulta indígena es la gran fortaleza de la participación ciudadana. Este logra involucrar a las comunidades, en relación a sus características socioculturales y de vinculación con el territorio (Fuenzalida y Quiroz 2012), buscando cierto consenso o acuerdos con ellos. Desde una lógica normativa, existe un intento por educar a las comunidades sobre los alcances de las actividades, ofreciéndoles información y respuestas técnicas ante preocupaciones relativas a los proyectos propuestos, incluyéndose al proceso evaluativo (Reyes y Ríos 2016). Sin embargo, este procedimiento sigue teniendo un aspecto meramente informativo y de consulta, buscando capacitar a las comunidades indígenas para que realicen observaciones ambientalmente fundamentadas, de esta manera, se mantiene la dinámica de las observaciones anteriormente descritas. A pesar de perpetuar la diferencia de información técnica y de oportunidades con respecto al titular, el reglamento permite adaptarse a la cultura indígena, asegurándose de que formen parte del proceso y logrando informar y capacitar comunidades con una cultura propia.

En términos teóricos, el concepto de participación ciudadana tiene arraigada la idea del empoderamiento de los sujetos y del grado de influencia que ellos pueden ejercer en los procesos de toma de decisión (Cunill 1991). La participación ciudadana es un proceso continuo y propositivo, individual o colectivo, donde se busca integrar a la toma de decisión, fiscalización y ejecución de las acciones públicas y privadas, afectando lo político, económico, social y ambiental (Llancar 2008). Bajo esta mirada, el procedimiento ex ante tiene la capacidad de integrar en el proceso de toma de decisión solo a un nivel informativo y de consulta. En otras palabras, existe un canal de retroalimentación, pero este no permite a los ciudadanos tener un grado de influencia asegurado. Así, haciendo alusión a la matriz de participación ciudadana (IAP2 2007), se considera el modo de consulta como un grado donde no existen las condiciones suficientes para que se tome en cuenta el punto de vista de los participantes. En este sentido, existe una forma de recibir opiniones y comentarios, se informa y se da importancia a las preocupaciones de la ciudadanía, existen reuniones con los órganos del Estado y se realizan grupos focales (comunidades indígenas), características que se relacionan con el nivel de consulta que se asegura dentro del procedimiento de evaluación ambiental.

Como consecuencia de lo analizado, es posible concluir que los espacios de participación ciudadana en Chile tienen serios problemas, sobre todo con los vacíos institucionales identificados. Los estrechos y tardíos espacios de participación reducen notablemente la capacidad de maniobra en materia ambiental, donde se ha perpetuado una participación informativa y consultiva, con espacios de reclamación restringidos. 


\section{ANÁLISIS UNIVARIADO}

De los $314 \operatorname{casos}^{10}$ observados - proyectos que incorporan mecanismos de participación ciudadana en el proceso de evaluación del SEIA- 67,2\% $(n=$ 211) corresponden a EIA y $32,8 \%(n=103)$ a DIA. Estas 314 observaciones representan solo un $4,95 \%$ del total de proyectos presentados $(n=$ $6.334)^{11}$, por lo tanto, los proyectos que han incorporado mecanismos de participación ciudadana constituyen una proporción pequeña.

Como muestra el Gráfico 1, al momento de establecer la distribución de casos por sector productivo, se puede identificar que los proyectos de energía concentran la mayor cantidad, con un $45,9 \%$. En segundo lugar, muy por debajo, se encuentran los proyectos de saneamiento ambiental, con un $17,5 \%$, seguido por los proyectos de minería, con $14 \%$. El resto de proyectos identificados no logran superar el $5 \%$ de los casos.

Con respecto a lo anterior, al momento de establecer la distribución regional de estos sectores, como se muestra en el Gráfico 2, los proyectos de energía concentran su máxima cantidad de casos en la Octava Región, con un $16,7 \%$, seguido por la Novena y la Tercera Región. Por otro lado, los proyectos de minería se concentran en gran medida en la zona norte de Chile, en la Tercera y Segunda Región, con un 34,1\% y un 27,3\%, respectivamente. Mientras que los proyectos de saneamiento ambiental predominan en la Séptima Región, con un 28,1\%.

10 Fueron excluidos todos aquellos proyectos EIA con estados de calificación no admitidos a tramitación, y en algunos casos, desistidos, cuando esta no fue la calificación final del proyecto. Es decir, cuando fueron presentados nuevamente al sistema.

11 Fueron eliminados los proyectos no admitido a tramitación, para equiparar las cifras con los proyectos con participación ciudadana. Si estos proyectos fueran incorporado, la cifra alcanzaría los 7.603 casos. 
Gráfico 1: Proyectos con participación ciudadana distribuidos por sector productivo $(N=314)$

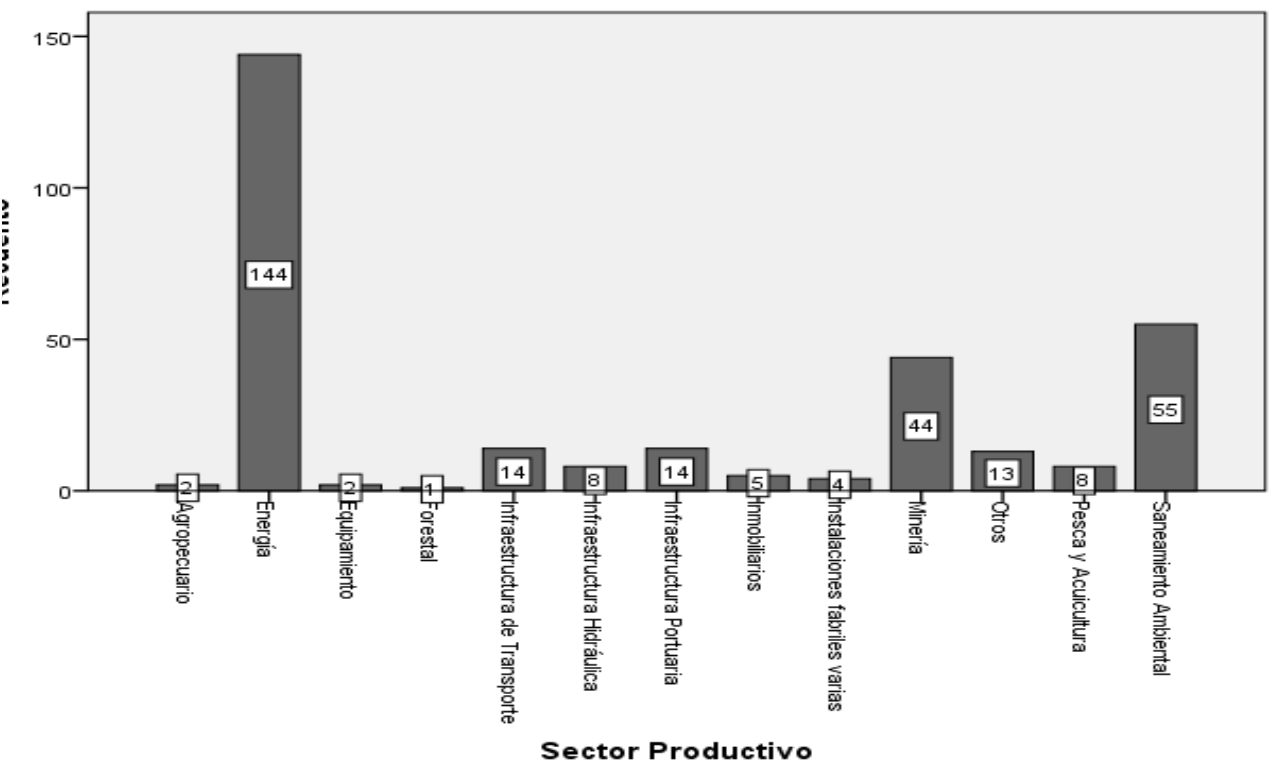

Fuente: Elaboración propia con base en datos solicitados a través de la Ley de Transparencia al SEIA.

Gráfico 2: Gráfico de Sector Productivo y Región $(N=243)$

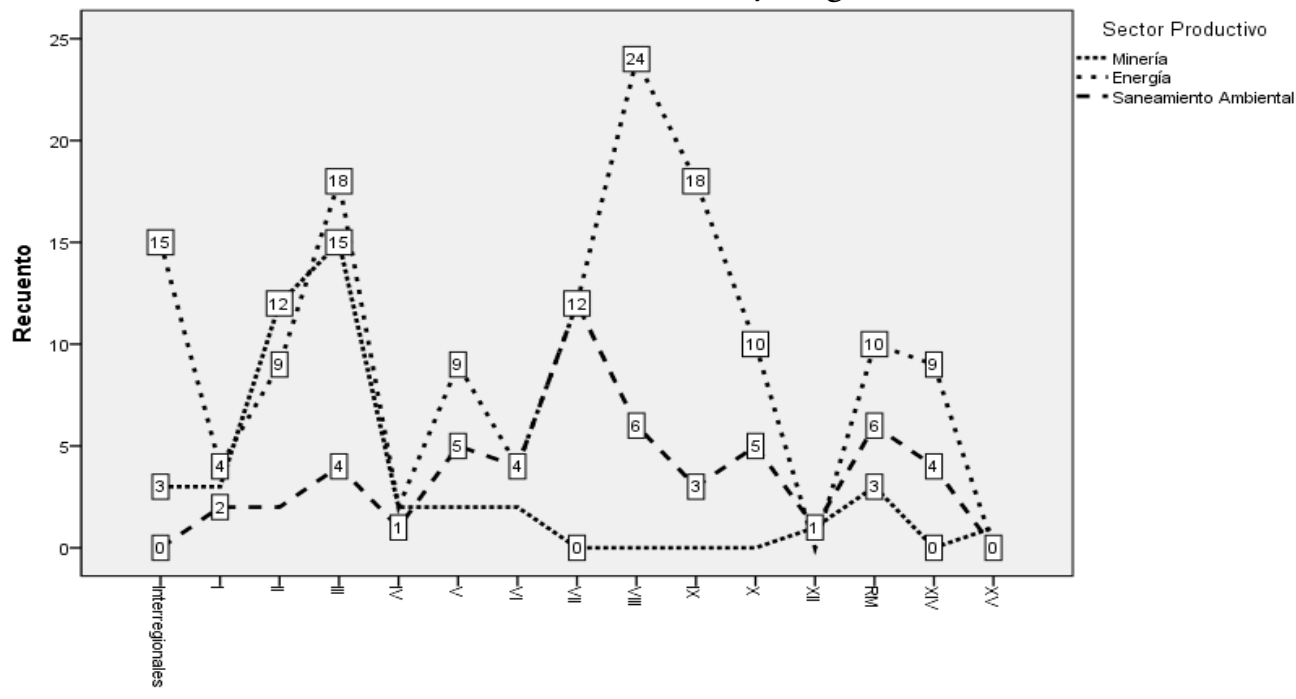

Región

Nota: Fueron excluidos proyectos cuyo sector productivo es inferior al $5 \%$. Fuente: Elaboración propia con base en datos solicitados a través de la Ley de Transparencia al SEIA. 
En relación a los estados de calificación, como se observa en la Tabla 2, del total de proyectos que han incorporados mecanismos de participación, la mayor cantidad de casos identificados corresponden a proyectos aprobados. En segundo lugar, se encuentran los proyectos en calificación, es decir, proyectos que aún no cuentan con una resolución definitiva. Seguidos, en tercer lugar, por los proyectos desistidos, caracterizados por ser retirados del sistema por los propios titulares. Por otro lado, muy por debajo a las cifras anteriores, se encuentran los proyectos no calificados y rechazados.

En vista de lo anterior, es posible establecer que la mayoría de los casos estudiados, que incorporan mecanismos de participación ciudadana, son aprobados por el sistema. Es decir, existe una tendencia favorable a las resoluciones positivas en desmedro de los otros estados de calificación.

Tabla 2: Estados de calificación en proyectos con mecanismos de participación ciudadana

\begin{tabular}{ccc} 
Estados & $N$ & Porcentaje \\
\hline Aprobado & 152 & $48,4 \%$ \\
Desistido & 36 & $11,5 \%$ \\
En Calificación & 105 & $33,4 \%$ \\
No calificado & 11 & $3,5 \%$ \\
Rechazado & 10 & $3,2 \%$ \\
Total & 314 & $100,0 \%$
\end{tabular}

Fuente: Elaboración propia con base en datos solicitados a través de la Ley de Transparencia al SEIA.

En términos de la utilización efectiva de los mecanismos durante el periodo en estudio, se logra identificar una media de 44,71 observaciones, con un rango de 0 a 1.482, y 4.65 actividades en promedio, con un rango de 0 a 57. De esta manera, como muestran los anexos 1 y 2 , en ninguno de los casos el gráfico permite asumir una distribución normal, existiendo varios valores inferiores a la curva, donde los proyectos caracterizados por una participación activa son escasos.

En relación al tiempo que dura la calificación de los proyectos $(n=204)^{12}$, medido en trimestres, se observa una media de 4,27, una moda de 3 y una mediana de 4. En este sentido, se puede establecer una gran concentración de proyectos con una demora igual o inferior a cuatro trimestres, con un rango de cero a 20. Al momento de comparar estos datos con aquellos

12 Fueron excluidos proyectos en calificación. 
calculados del total de proyectos presentados, donde el tiempo promedio de duración es de 1,38 trimestres, es posible señalar que una proporción alta de proyectos con mecanismos de participación ostentan tiempos calificación más extensos. A veces, incluso, estos superan los plazos establecidos por la ley. No obstante, en rigor, lo que sucede es que estos procedimientos suelen ser suspendidos, lo que aumenta tiempo del trámite, pero no necesariamente significa un incumplimiento de la norma.

Profundizando en el punto anterior, como muestra la Tabla 3, el tiempo de demora fue agrupado en cinco niveles. Al momento de comparar el tiempo de duración con los estados de calificación, se puede observar que los proyectos aprobados concentran su máxima cantidad de casos en el quinto grupo, con cuatro o más trimestres (un año o más), seguido decrecientemente por el resto de los grupos. De esta manera se puede observar una tendencia moderada baja y positiva. En el caso de los proyectos desistidos y rechazados, si bien no es posible establecer la existencia de asociación entre las variables, estas también se concentran fuertemente en el quinto grupo. De esta forma, se puede establecer que existe la posibilidad de que los mecanismos de participación retrasen los tiempos de demora de las resoluciones, ya sea en proyectos aprobados o rechazados.

Tabla 3: Estados de Resolución y Trimestres de Calificación $(N=204)$ Estados de calificación

\begin{tabular}{cccccc}
$\begin{array}{c}\text { Trimestres } \\
\text { agrupados }\end{array}$ & Aprobado & Desistido & $\begin{array}{c}\text { No } \\
\text { calificado }\end{array}$ & Rechazado & Total \\
\hline$<=0$ & - & $100,0 \%$ & $100,0 \%$ & $10,0 \%$ & $8,8 \%$ \\
1 & $5,3 \%$ & - & - & - & $3,9 \%$ \\
2 & $13,8 \%$ & - & - & - & $11,8 \%$ \\
3 & $25,0 \%$ & - & - & $20,0 \%$ & $21,1 \%$ \\
4 o + & $55,9 \%$ & - & - & $70,0 \%$ & $54,4 \%$ \\
Total & $100,0 \%$ & $100,0 \%$ & $100,0 \%$ & $100,0 \%$ & $100,0 \%$
\end{tabular}

Nota: Los proyectos en calificación fueron excluidos. Los proyectos de aprobados, tienen un $R$ de 0,314 ( $p<0,000)$; los proyectos desistidos, tienen un $R$ de -0,049 ( $p<0,483$ ); los proyectos no calificados, tienen un $R$ de -0,581 ( $<<0,000)$; por último, los proyectos rechazados, tienen un $R$ de 0,057 ( $p<0,420)$.

Fuente: Elaboración propia con base en datos solicitados a través de la Ley de Transparencia al SEIA. 
Finalmente, en cuanto a la inversión, se puede observar una situación similar. Al momento de analizar el nivel de inversión presupuestado de cada uno de los proyectos que incorporan mecanismos de participación, se puede identificar una media de US\$268.210.000, con una moda de US\$ 300.000 .000 y una mediana de US\$ 50.000.000. Ahora bien, al comparar estos datos con la cantidad total de casos, donde la media es de US\$ 37.970.000, destaca una gran diferencia en los montos de inversión a favor de los proyectos que incurren en algún tipo de participación ciudadana.

\section{ANÁLISIS BIVARIADO}

$\mathrm{Al}$ momento de establecer el nivel de relación entre las diversas variables ya estudiadas, como muestra la Tabla 4, se puede identificar, en primer término, la existencia de una asociación moderada-baja entre la cantidad de observaciones y actividades. En este sentido, a pesar identificar un nivel de linealidad bajo, es posible apreciar una relación positiva entre ambas variables. En otras palabras, las actividades podrían determinar un mayor número de observaciones, debido a que estas instancias tienen por finalidad capacitar a los ciudadanos para que logren enviar satisfactoriamente sus inquietudes y adecuarse a la evaluación.

Situación similar sucede al observar el nivel de asociación entre la cantidad de observaciones y el nivel de inversión y entre número de actividades y el nivel de inversión, alcanzando un nivel de relación moderado-bajo y moderado-alto, respectivamente. Bajo estos resultados, es posible plantear que mientras mayor sea la inversión de los proyectos, mayor sería la cantidad de actividades. Eventualmente, proyectos de mayor envergadura generaría una mayor atención de la ciudadanía.

Sucede un fenómeno distinto al momento de analizar la relación entre las observaciones y actividades con los tiempos de demora, donde se puede establecer un bajo nivel de relación en ambos casos. En este sentido, se puede establecer que la participación no logra explicar el aumento de los plazos en la evaluación, que como se señaló en el análisis univariado, tienden a ser más extensos. 
Tabla 4: Correlaciones de Observaciones, actividades, tiempo de demora (meses) e inversión

\begin{tabular}{cccc}
$\begin{array}{c}\mathrm{N}^{\circ} \mathrm{de} \\
\text { observaciones }\end{array}$ & $\begin{array}{c}\mathrm{N}^{\circ} \mathrm{de} \\
\text { actividades }\end{array}$ & Meses & Inversión \\
\hline
\end{tabular}

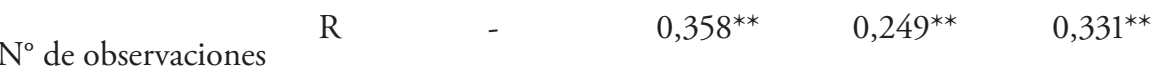

\begin{tabular}{|c|c|c|c|c|c|}
\hline & $\mathrm{R}^{2}$ & - & $0,128^{* *}$ & $0,062^{* *}$ & $0,110^{* *}$ \\
\hline \multirow{2}{*}{$\mathrm{N}^{\circ}$ de actividades } & $\mathrm{R}$ & $0,358^{* *}$ & - & $0,281^{* *}$ & $0,483^{* *}$ \\
\hline & $\mathrm{R}^{2}$ & $0,128^{* *}$ & - & $0,079^{* *}$ & $0,233^{* *}$ \\
\hline \multirow{2}{*}{ Meses } & $\mathrm{R}$ & $0,249^{* *}$ & $0,281^{* *}$ & - & $0,076^{* *}$ \\
\hline & $\mathrm{R}^{2}$ & $0,062^{* *}$ & $0,079^{* *}$ & - & $0,006^{* *}$ \\
\hline \multirow[t]{2}{*}{ Inversión } & $\mathrm{R}$ & $0,331^{* *}$ & $0,483^{* *}$ & $0,076^{* *}$ & - \\
\hline & $\mathrm{R}^{2}$ & $0,110^{* *}$ & $0,233^{* *}$ & $0,006^{* *}$ & - \\
\hline
\end{tabular}

Nota: ** La correlación es significativa al nivel 0,$01 ;{ }^{*}$ La correlación es significativa al nivel 0,05 .

Fuente: Elaboración propia con base en datos solicitados a través de la Ley de Transparencia al SEIA.

\section{CONCLUSIONES}

En la política ambiental el SEIA ha sido el instrumento insigne de gestión ambiental, con una competencia técnica y una capacidad de coordinación importante en la materia. Sin embargo, esta se ha quedado atrás en términos de la inclusión de componentes sociales. Como consecuencia, en abril del año 2015, la presidenta Bachelet creó la Comisión Asesora Presidencial para el estudio de un nuevo SEIA. En su mandato, se busca una respuesta al nuevo escenario sociocultural y económico, con un procedimiento más expedito, pero que al mismo tiempo le diera un marco de certeza jurídica a los titulares, a la comunidad y a los distintos servicios públicos (Durán et al. 2016). Con base a lo anterior, existe una clara búsqueda por la eficiencia de plazos, pero no se hace mayor referencia a los mecanismos de participación ciudadana, problema que se ha podido constatar en la presente investigación.

En este sentido, podría ser importante mencionar un nuevo concepto dentro del presente esquema, el capital social. Este término está asociado 
a un conjunto de características intangibles de una comunidad, como la capacidad de trabajar de manera colaborativa en función de objetivos o metas compartidas en función del bien común (Maldonado 2000). Esta condición requiere de ciudadanos preparados y capaces de incidir en las decisiones, tomando conciencia de las implicancias de sus posturas y demandas (Berdichevsky y Sepúlveda 2016).

Sin embargo, tomando en cuenta los datos expuestos en este artículo, se observa una falta de interés aparente en la participación de espacios formales. En este sentido, la participación ciudadana parece encontrarse en un estado pasivo. Los individuos, notando serias limitaciones en las consideraciones de opiniones, deciden utilizar canales informales o no institucionalizados, tales como las paralizaciones, para hacer frente a las injusticias percibidas.

Por otra parte, aunque parcialmente, es posible sostener que los procedimientos de evaluación de impacto ambiental están orientados a la aprobación de los proyectos. Al respecto, cabría preguntarse por los factores que dan cabida a este fenómeno.

Finalmente, a pesar de que existen casos donde la participación ha sido abrumadora por parte de los ciudadanos -aunque constituyen una minoría-, existen limitaciones del proceso que no permiten incidir en las decisiones finales. Al respecto, un punto crítico es la incapacidad, tanto del SEIA como de los ciudadanos, para priorizar o establecer identidades y valores ambientales a los territorios. Este elemento requiere ser considerado en el proceso de evaluación ambiental, con la finalidad de prevenir posibles focos de conflictos originados por la incompatibilidad territorial de los proyectos (Durán et al. 2016). A esto también se suma el tardío espacio formal de la participación ciudadana, que comienza una vez concluido el diseño de ingeniería, por lo que la posibilidad de los ciudadanos de incidir en la elaboración del proyecto es demasiado estrecha en términos de la idea, la prefactibilidad y factibilidad de las inversiones (Berdichevsky y Sepúlveda 2016). Es durante estas etapas donde los ciudadanos tienen mayores oportunidades de incidir en las decisiones, y es aquí también donde la resistencia a los cambios es menor, por lo que la reflexión apunta a repensar estos espacios de discusión y a incluir ciertos elementos que no han sido considerados dentro de la evaluación, cuya consideración podría tener un impacto significativo en la legitimidad del proceso.

Hoy, Chile enfrenta una encrucijada producto del masivo cuestionamiento ciudadano a las decisiones ambientales y a la creciente judicialización de las RCA. Casos como la central hidroeléctrica Hidroaysén o el proyecto minero Pascua Lama, confirman que la sociedad chilena ha sido más 
exigente cuando el caso lo amerita, donde también se han visto actores más preparados para activar contrapesos. Así, los ciudadanos prefieren hacer uso de una participación directa de baja institucionalización (PNUD 2014). En este sentido, según Hernández y Sazo (2015), siete de los diez proyectos con mayor inversión en Chile se encuentran hoy paralizados, gracias a una activa resistencia de grupos organizados. Finalmente, terminan siendo estos tipos de manifestaciones ciudadanas las que tienen más adherentes, y no los mecanismos formales que el Estado ha puesto a disposición.

Así, el estudio del rol de los mecanismos de participación ciudadana en los procesos de decisión, tanto en su creación como en su desarrollo, es fundamental para un mejor desempeño de la democracia. En este sentido, la transparencia, la consulta y el involucramiento ciudadano en las decisiones públicas permiten obtener un grado alto de legitimidad a lo largo de estos procesos.

Este trabajo mostró que, en la actualidad, el rol que se lo otorga a los ciudadanos en la protección ambiental no es significativo, y la participación no está asegurada para generar una real influencia en las decisiones de la autoridad. 


\section{REFERENCIAS}

Alarcón, L., Gómez, I. y Rodríguez, L. (2004). Actores y Participación Ciudadana en el Actual Escenario Sociopolítico. Frónesis, 11 (3), 65120.

Berdichevsky, P. y Sepúlveda, C. (2016). Desafíos de una Política Pública en base a Dos Décadas de Aprendizaje. Espacio Público, 27, 2-51.

Bermúdez J. (2010). Acceso a la Información Pública y la Justicia Ambiental. Revista de Derecho Pontificia Universidad Católica de Valparaíso, 34, 571-596. . (2014). Fundamentos de Derecho Ambiental. Valparaíso: Ediciones Universitarias de Valparaíso.

Boettiger, C. (2010). Nueva Institucionalidad Ambiental. Actualidad Jurídica, 22, 429-454.

Carmona, R. (2012) Políticas Públicas y Participación Ciudadana en la Esfera Local. Análisis y Reflexiones a la Luz de la Experiencia Argentina Reciente. Revista Chilena de Administración Pública, 19, 169-185.

Cunill, N. (1991). Participación Ciudadana: Dilemas y Perspectivas para la Democratización de los Estados Latinoamericanos. Caracas: Centro Latinoamericano de Administración para el Desarrollo.

Durán, V., González, G., Ríos, E., Sepúlveda, C., Schmitt, L. y Wagner, R. (2016). Del Conflicto al Diálogo: Cómo Avanzar hacia un Sistema Eficiente de decisiones Ambientales Participativas. Espacio Público, 8, 3-92.

Estenssoro, F. (2009). Medio Ambiente e Ideología. La discusión Pública en Chile, 1992-2002. Santiago: Adriadna.

Fuenzalida, M. y Quiroz, R. (2012). La Dimensión Espacial de los Conflictos Ambientales en Chile. Polis, Revista Latinoamericana, 11 (31), 157-168.

González, G. (2011). Fortalecimiento del Sistema de Evaluación de Impacto Ambiental de Chile: Lecciones de la Legislación Internacional. Disponible en https://www.nrdc.org/laondaverde/international/files/seiareport. pdf [02-02-2016]. 
Hernández, C. y Sazo, D. (2015). Movilización y Resistencia Verde: Los conflictos Socioambientales en Chile, 2000-2013. Revista de Gestión Pública, 4 (2), 193-227.

International Association for Public Participation (IAP2) (2007). Spectrum of Public Participation. Disponible en http://c.ymcdn.com/sites/www. iap2.org/resource/resmgr/imported/IAP2\%20Spectrum_vertical.pdf [12-01-2016].

Lechner, N. (1991). El Ciudadano y la Noción de lo Público. Revista Leviatán, 43/44, 107-116.

Llancar, C. (2008). Sociedad Civil y Participación Ciudadana - Cómo los Actores Sociales se Hacen Parte de las Decisiones. Interaçôes Campo Grande, 9 (2), 181-188.

Maldonado, C. (2000). Reseña de "Making Democracy Work". Estudios Sociológicos, 18 (3), 737-739.

Macpherson, C. B. (2003). La Democracia Liberal y su Época. Madrid: Editorial Alianza.

Mirosevic C. (2011). La Participación Ciudadana en el Procedimiento de Evaluación de Impacto Ambiental y las Reformas Introducidas por la Ley No 20.417. Revista de Derecho Pontificia Universidad Católica de Valparaiso, 36, 281-323.

Montero, M. (1996). La Participación: Significados, Alcances y límites. En Hernández, E. (Ed.), Participación: Ámbitos, Retos y Perspectivas. Caraca: CESAP.

Organización para la Cooperación y el Desarrollo Económico (OCDE). (2005). Evaluaciones de Desempeño Ambiental Chile. Santiago: Comisión Económica para América Latina y Chile.

Olivares, A. (2010) El Nuevo Marco Institucional Ambiental en Chile. Revista Catalana de Dret Ambiental, 1 (1), 1-23.

Oro, L. (2014). Derecho Político: Aspectos de la Conflictividad. Anales de Facultad de Ciencias Jurídicas y Sociales, 11 (44), 162-170.

Programa de Naciones Unidas para el Desarrollo (PNUD). (2014). Ciudadanía Politica: Voz y Participación Ciudadana en América Latina. 
Madrid: Programa de las Naciones Unidas para el Desarrollo.

Reyes, F y Ríos, E. (2016). Participación Ciudadana en Proyectos de Inversión: Lecciones desde la Experiencia Internacional. Espacio Público, 26, 2-59.

Sepúlveda, C. y Rojas, A. (2010). Conflictos Ambientales y Reforma Ambiental en Chile: Una Oportunidad Desaprovechada de Aprendizaje Institucional sobre Participación Ciudadana. Revista Ambiente y Desarrollo de CIPMA, 24 (2), 15-23.

Sierra, L. (2008). Reforma de la Institucionalidad Ambiental: Problemas y Oportunidades. Estudios Públicos, 111, 57-101.

Vargas, V. (1999). Ciudadanías Globales y Sociedades Civiles Globales. Pistas para su Análisis. Disponible en www.ico.org.uy/mercosur/forum/ vargas.html [09-01-2016].

Villarroel R. (2014). Ética del Desarrollo, Democracia Deliberativa y Ciudadanía Ambiental. El Desafío Global de la Sustentabilidad. Revista de Filosofía, 70, 161-174.

Zelada, S. y Park, J. (2013). Política Ambiental Chilena y Política Indígena en la Coyuntura de los Tratados Internacionales (1990-2010). Polis, Revista Latinoamericana, 12 (35), 555-575.

Decretos y leyes

Decreto Supremo 40, Aprueba Reglamento del Sistema de Evaluación de Impacto Ambiental. Diario Oficial de La República de Chile, 12 de agosto de 2013, Santiago, Chile.

Ley 19.273, Ley Establece Normas sobre Protección, Fomento y Desarrollo de los Indígenas, y Crea la Corporación Nacional de Desarrollo Indígena. Diario Oficial de La República de Chile, 5 de octubre 1993, Santiago, Chile.

Ley 19.300, Ley Sobre Bases Generales del Medio Ambiente. Diario Oficial de La República de Chile, 9 de marzo de 1994, Santiago, Chile.

Ley 19.880. Diario Oficial de La República de Chile, 29 de mayo de 2003, Santiago, Chile. 
Ley 20.173. Diario Oficial de La República de Chile, 27 de marzo de 2007, Santiago, Chile.

Ley 20.285, Ley Sobre Acceso a la Información Pública. Diario Oficial de La República de Chile, 20 de agosto de 2010, Santiago, Chile.

Ley 20.417. Diario Oficial de La República de Chile, 12 de enero de 2010, Santiago, Chile.

Ley 20.500, Ley Sobre Asociaciones y Participación Ciudadana en la Gestión Pública. Diario Oficial de La República de Chile, 4 de febrero de 2011, Santiago, Chile.

Recibido: 03-09-2017

Aceptación de la versión final: 27-12-2017 


\section{ANEXOS}

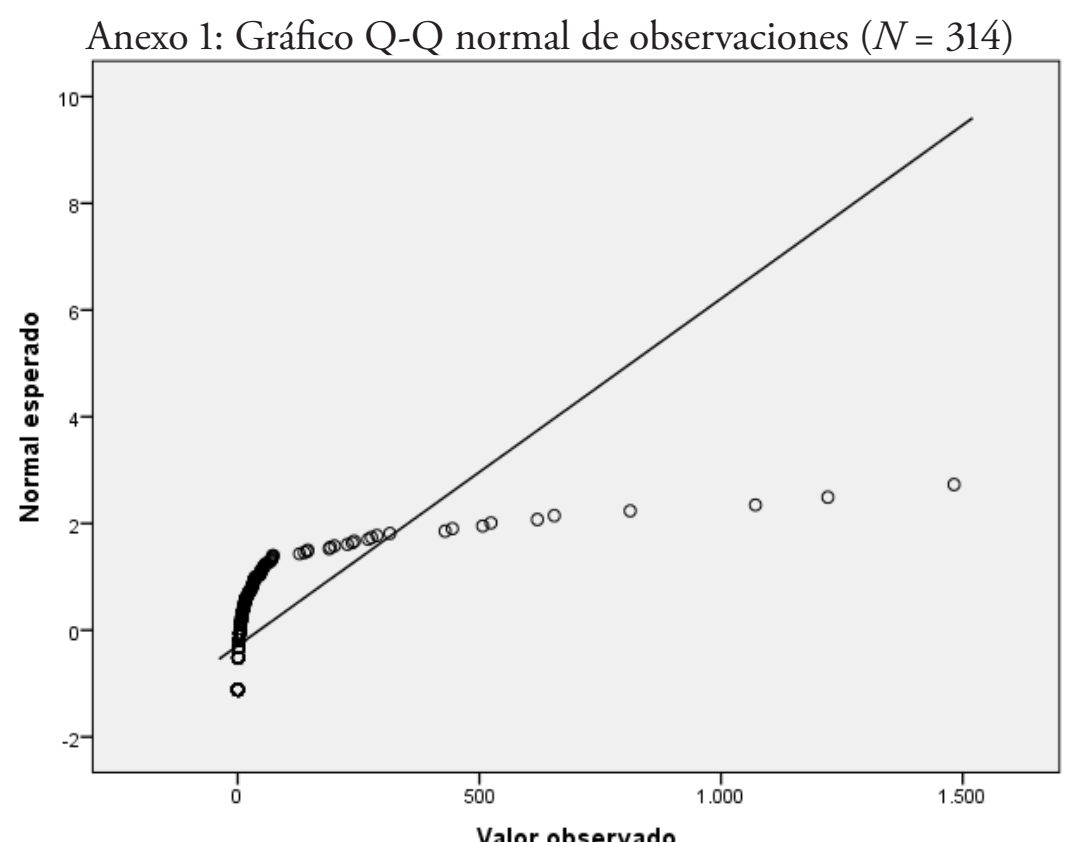

Nota: $\Sigma=14.039$ observaciones; $M=44,71$ observaciones; $M d n=5$ observaciones; $M d a=0$ observaciones. La curva no permite asumir una distribución normal en las observaciones (Kolmogorov Smirnov $=0,000$ ). Fuente: Elaboración propia con base en datos solicitados a través de la Ley de Transparencia al SEIA. 
Anexo 2: Gráfico Q-Q normal de actividades $(N=314)$

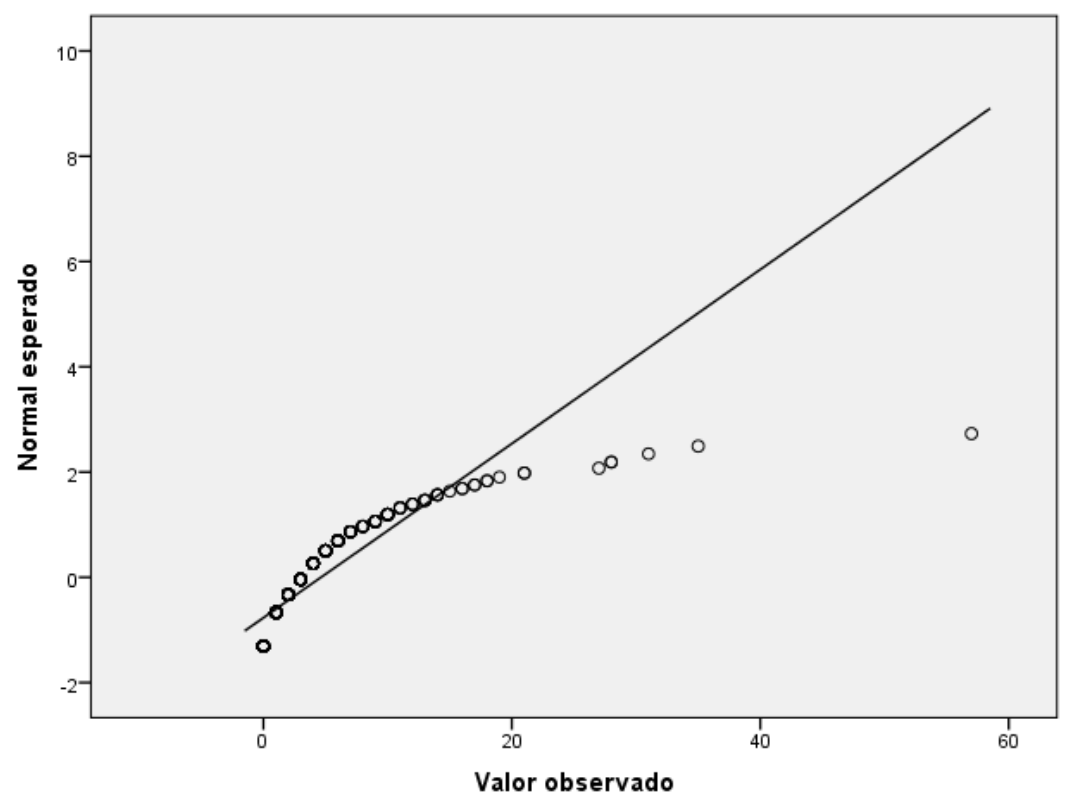

Nota: $\Sigma=1.459$ actividades; $M=4,65$ actividades; $M d n=3$ actividades. La curva no permite asumir una distribución normal en las actividades (Kolmogorov Smirnov =0,000). Fuente: Elaboración propia con base en datos solicitados a través de la Ley de Transparencia al SEIA. 